Supporting Information

\title{
Structure-dependent photoluminescence of europium (III) coordination oligomeric silsesquioxane: synthesis and mechanism
}

Ming Li, a, b Chengyu Wang, ${ }^{a}$ Di Wang ${ }^{*_{a}}$ and Jian $\mathrm{Li}^{{ }^{* a}}$

${ }^{a}$ Material Science and Engineering College Northeast Forestry University, Hexing Road 26, Harbin 150040, P.R. China.

${ }^{b}$ Key Laboratory of Bio-based Material Science and Technology of Ministry of Education, Northeast Forestry University, Hexing Road 26, Harbin 150040, P.R. China.

$\square$ Corresponding author: E-mail: diwang1030@nefu.edu.cn (D. Wang), nefujianli@163.com (J.Li).

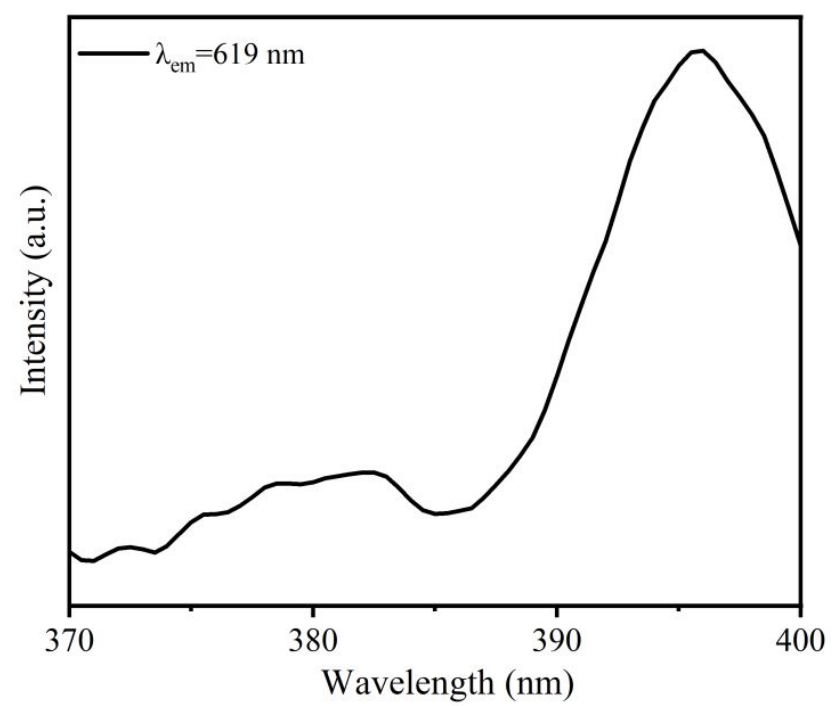

Figure S1. Excitation spectra of the europium (III) coordination oligomeric silsesquioxane

(a)

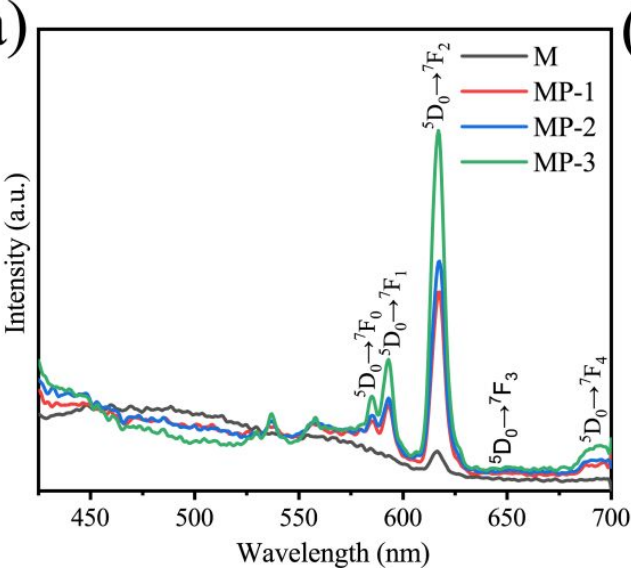

(b)

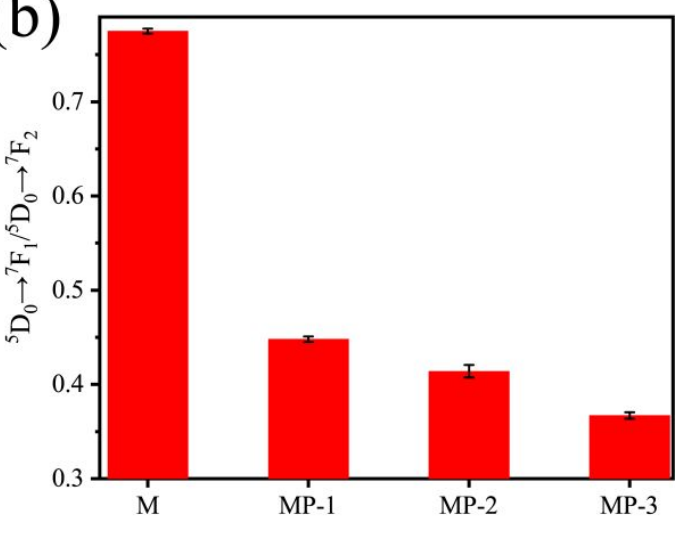

Figure S2. Emission spectra of M and MP (a) and intensity ratio value of ${ }^{5} \mathrm{D}_{0} \rightarrow{ }^{7} \mathrm{~F}_{1} /{ }^{5} \mathrm{D}_{0} \rightarrow{ }^{7} \mathrm{~F}_{2}$ (b). 


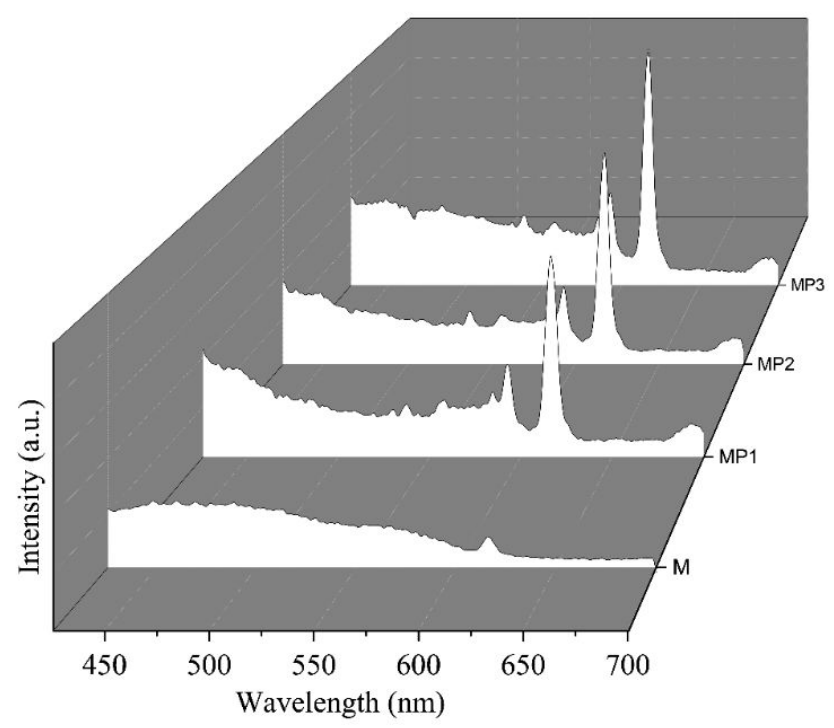

Figure S3. Emission spectra of M and MP (1:4) with different sol-gel reaction time (48h (MP1), $60 \mathrm{~h}$ (MP2), 72h (MP3) (optimum condition)) at $40^{\circ} \mathrm{C}$

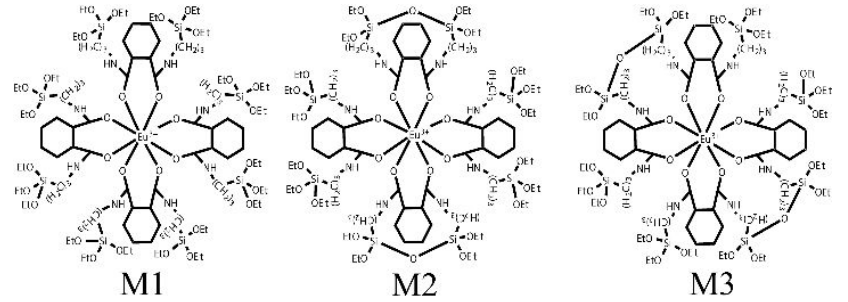

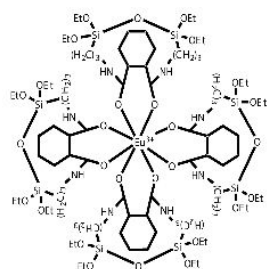

M4

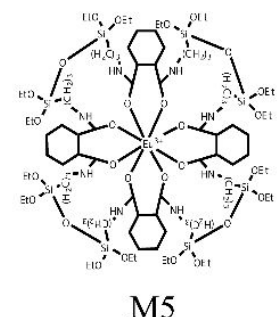

M5

Figure S4. Theory geometry structures of complexes

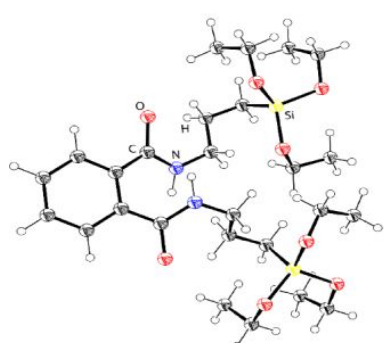

LSi

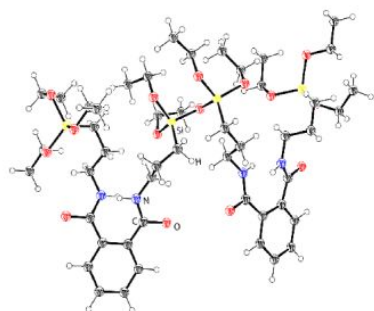

LSi-2

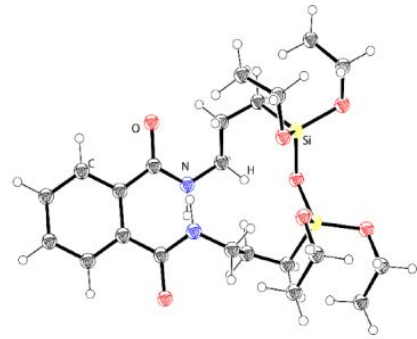

LSi-1

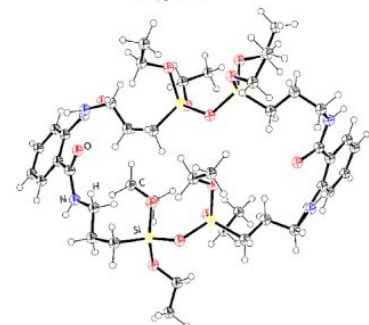

LSi-3 
Figure S5. Theory geometry structures of ligands

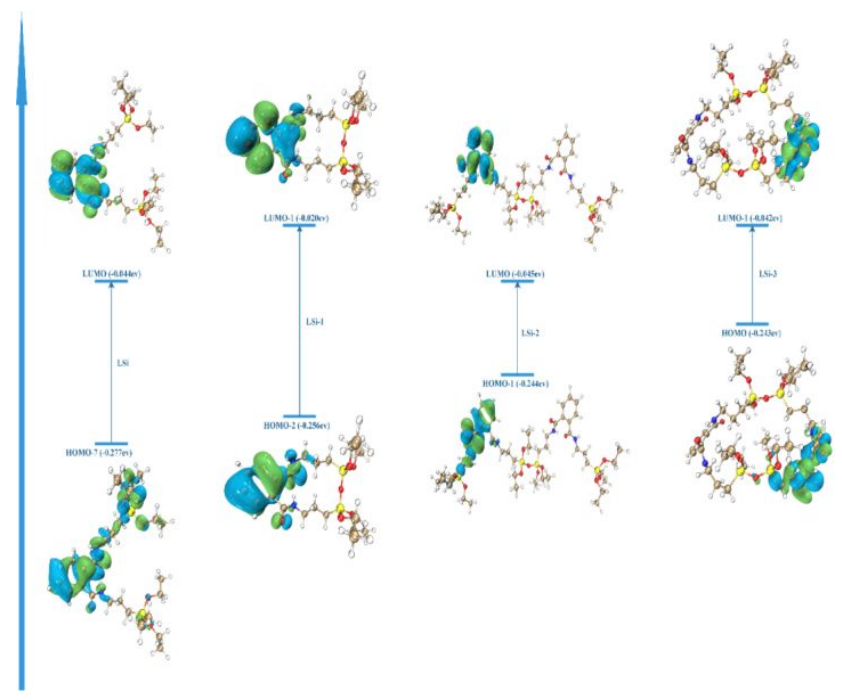

Figure S6. The occupied and unoccupied orbitals contributing mostly to the singlet excitation of L, LSi, LSi-1, LSi-2 and LSi-3 